Results 15 babies met inclusion/exclusion criteria. All babies were $37-40$ weeks gestation. $8 / 15$ were male, mean birth weight was $3.36 \mathrm{~kg}$ and mean maternal age was 31.9 years. 10/15 (66\%) infants had evidence of bacteria on FISH. Of these, RT-PCR was positive in only 1 . Positive FISH counts ranged from 2.2 to $41.8 * 10^{4}$ cells/g with the mean of positive samples being $15.4 * 10^{4}$ cells/g. (Limit of detection for automated counting is $10^{6}$ cells $\left./ \mathrm{g}\right)$. Cell counts were too low to allow formal diversity analysis. Amplification by RT-PCR was not possible despite positive spiked samples demonstrating the feasibility of reaction. Three babies were dominated by a single family, either Enterobacteriaceae or Enterococcaceae. The others contained 2-5 genera. Bifidobacterium, Enterobacteriaceae and BacteroidesPrevotella were the most dominant bacteria identified. There was no association between rupture of membrane duration, time to passage of meconium or time to lab with bacterial counts.

Conclusion Evidence of bacteria in first-pass meconium samples from healthy, vaginally-delivered, breastfed term infants is scant with only two-thirds having demonstrable bacteria at levels too low for automated counting. Bacterial RT-PCR failed to amplify 9/10 FISH-positive samples. This study suggests that gut bacterial diversity is extremely limited at birth and supports the hypothesis that the neonatal gut is sterile and colonised rapidly thereafter.

\section{G117(P) IMPACT OF AN EARLY WARNING SYSTEM FOR NURSING OBSERVATIONS IN AT-RISK NEONATES IN THE POST NATAL WARDS}

1,2T Pillay, ${ }^{1} \mathrm{M}$ Doodson, ${ }^{1} \mathrm{M}$ Nash, ${ }^{2} \mathrm{R}$ Moore. ${ }^{1}$ Neonatal Unit, New Cross Hospital, Royal Wolverhampton Hospitals Trust, Wolverhampton, UK; ${ }^{2}$ Staffordshire, Shropshire and Black Country (SSBC) Newborn Network, West Midlands, UK

\subsection{6/archdischild-2015-308599.116}

Aim In line with national recommendations for similar systems in other disciplines, the SSBCNN implemented an Early Warning System (EWS) for nursing observations in the at-risk neonate on the post natal ward. The impact on nursing workload, utility and efficiency of this EWS was audited, as part of an initiative to understand trigger events for intervention in at-risk babies.

Method Case notes of 300 neonates following implementation of EWS, and 240 predating its implementation were reviewed. In the latter a retrospective EWS chart was completed using data extracted from notes. Early warning triggers were observations falling outside the acceptable colour coded range. Observations were analysed in at-risk neonates with meconium stained liquor, maternal history of prolonged rupture of membranes, maternal group B Streptococcus infection and small for gestational age. Feedback from 56 nurses in the network was obtained using a structured questionnaire.

Results A nursing observation that was intended to trigger an intervention was reached in 261(48.3\%) at-risk babies. Intervention was recorded in $25 \%$; in the remainder, no action was taken, or none documented. Low temperature $\left(<36.5^{\circ} \mathrm{C}\right)$ was the commonest trigger. In subgroup analyses, $41.8 \%$ of hypothermia was recorded within the first $2 \mathrm{~h}$ of birth. $1.7 \%$ babies were admitted to the neonatal unit in response to trigger observations.

Fewer trigger observations were noted in the post-implementation group $(144 / 240$ vs $117 / 300 ; p=<0.01)$. This was specifically significant for hypothermia $(125 / 240$ vs $88 / 300 ; p=$ $<0.001)$. The EWS did not increase the number of observations per category of stable at-risk neonates. The duration of hospital stay was 10-14 h shorter post-implementation of EWS in those babies delivered vaginally. Nursing feedback was positive in all; $82 \%$ had used the chart in $>20$ with $30 \%$ having used it in over 50 babies each, prior to responding.

Conclusion The implementation of EWS was associated with a reduction in trigger observations, implying improved neonatal clinical condition, specifically temperature control. This improvement infers greater attention to detail accompanying systematic recording of observations; however, a direct association between the two cannot be proved. The EWS did not increased nursing workload, and contributed to increased efficiency, measured through shorter hospital stays for those born vaginally.

\section{G118(P) LIVING WITH CHRONIC LUNG DISEASE (CLD); CHILDREN AND PARENTS PERSPECTIVES}

${ }^{1,2} \mathrm{~L}$ Bray, ${ }^{1,3} \mathrm{NJ}$ Shaw, ${ }^{4} \mathrm{~J}$ Snodin. ${ }^{1}$ Evidence-Based Practice Research Centre, Edge Hill University, Ormskirk, UK; ${ }^{2}$ Children's Nursing Research Unit, Alder Hey Children's NHS Foundation Trust, Liverpool, UK; ${ }^{3}$ Neonatal Unit, Liverpool Women's Hospital, Liverpool, UK; ${ }^{4}$ Faculty of Health and Social Care, Edge Hill University, Ormskirk, UK

\subsection{6/archdischild-2015-308599.117}

Introduction Caring for a premature baby who is oxygen dependant may result in short-term parental fatigue, anxiety, depression and restrictions to lifestyle, however long-term effects have not been reported.

Aim To investigate children and their parents' longer-term experiences of living with CLD.

Methods This was an exploratory qualitative study which collected interview data from children with CLD aged between 6 and 15 years of age $(n=10)$ and their parents $(n=12)$. Families were recruited from a children's hospital in the UK. Data were analysed inductively and thematically.

Results Children and their parents described that CLD was 'easier to live with as you get older'; this was in some cases due to symptoms becoming less severe but was also influenced by the strategies and expertise acquired in managing the condition. CLD was contextualised against other disabilities caused by prematurity and the uncertainty and fear which had overshadowed the first few years of the child's life. Parents had gained proficiency in accessing health services which demonstrated competency in dealing with CLD and bypassing those seen as less helpful. Parents felt anxious when they devolved responsibility for managing their child's condition to others, such as schools, and when they thought about their child's future. Children's concerns related to when CLD made them feel different to their friends by causing them to have to step-back, sit out and miss out on activities or when they were ostracised due to their short stature, respiratory symptoms or fatigue. Despite expertise in recognising and managing symptoms, children and their parents discussed a poor understanding of the nature of the condition and this influenced how they described CLD to friends, family, school and outside agencies. Many chose to call the condition asthma; the descriptor of CLD was seen as unhelpful and implied a contagious condition.

Conclusions Children and parents' accounts were mainly positive with CLD becoming easier to live with and manage over time. Ongoing concerns related to managing CLD outside the family, situations causing children to feel different and a poor understanding of the nature of CLD. 